\title{
Towards Cognitive Communication and Other Applications of Cognitive Computing
}

\author{
Andy E. Williams, Nobeah Foundation, Nairobi, Kenya
}

\begin{abstract}
The term cognitive communications has been used to describe "human-centric" communication systems that adapt to different behaviors, expectations and preferences. This paper explores a more general use of the term by attempting to enumerate all communication functions that might benefit through being executed by systems of individual or collective cognition. Systems of individual cognition might be represented by intelligent agents (based on some subset of the functionality suggested to be required for Artificial General Intelligence) with the capacity to change any property of communication. Communication functions executed by such systems optimize individual outcomes. Systems of collective cognition might be represented by collective intelligence solutions (based on some subset of functionality suggested to be required for General Collective Intelligence) with the capacity to enable such intelligent agents to self-assemble into communication networks using any combination of network topology, protocols, spectrum or other properties. Communication functions executed by such systems optimize collective outcomes. From this perspective, cognitive communication is explored as a specific case that might be generalized to apply to any number of other sectors, such as cognitive power generation and distribution, cognitive agriculture, cognitive healthcare, etc.
\end{abstract}

\section{Keywords}

cognitive communication systems, cognitive computing, General Collective Intelligence, Artificial General Intelligence, intelligent agent

\section{Introduction}

Where the term "cognitive radio" describes a system that automatically adapts to use the best available band for communication, recent work in cognitive communication explores how cognitive computing might address any problem of communication whatsoever [4], [5], [6], [7]. In a GCI orchestrated cognitive communication" process, intelligent agents based on some subset of AGI might work behalf of each user to negotiate the best available physical connection, electromagnetic spectrum, protocol at each protocol layer, network topology, and every other function of Internetworking and telecommunication [8], [9]. And GCI might orchestrate that cooperation to adaptively learn which implementation of each function is most fit in each context from all possible occurrences of Internet and telecommunication use. The usefulness of doing so would be expected to be increased sustainability across the entire product life-cycle, an increase in affordable access to communication, and an increase in quality of communication at each level of affordability. Because network changes or upgrades resulting in inoperable devices that have to be discarded would be expected to disappear. A conceptual example of why this sustainability is not reliably achievable without GCI has been explored in consumer electronics (headsets) [11]

\section{Individual and Collective Cognitive Computing}

Since potentially suitable models of AGI [1] and GCI [2], [3] have just recently been defined, but have not yet been implemented, the question arises why an AGI and GCI based framework for navigating computing methods should be considered at this early stage, and how the high-level properties of such a framework can be objectively validated and therefore add concrete value rather than being mere speculation. As for why consider such a framework, the simply answer is that doing so creates the potential to exponentially increase outcomes targeted by any computing methods, where doing so can be demonstrated to be outside the capacity of any human intellect to reliably achieve otherwise. As explored in the domain of sustainable housing design, any design process faces limits to the complexity it has the capacity to navigate [10]. Similarly, in the computing domain, where computing methods designed by any individual human without AGI, or by any group without GCI can only define the problems that can fit inside human cognition, and can only solve those problems with 
the solutions that are discoverable within human cognition, the combination of AGI and GCI can be demonstrated to have the capacity to reliably generate an exponential increase in general problem-solving ability [13]. Measured in impact on any outcome, such as the outcomes targeted through the design of computing methods, this can be expected to drive an exponential increase in impact on those outcomes.

AGI creates the potential to explore the fitness of every combination of different technologies in implementing every computing operation, and AGI creates the potential to explore interactions between computation operations that are much higher order (much more complex) than currently possible, and to do so at vastly greater speed and scale, in order to achieve vastly greater impact on any targeted outcomes of computation. GCI creates the potential to collectively store information about which combination of different technologies is most fit in implementing every computing operation, so that the computing operations executed by any one individual benefit from intelligence gained from the execution of any computing operation by any other individual. GCI also creates the potential to enable higher order interactions between computation operations that orchestrate group processes, and to do so at vastly greater speed and scale, in order to achieve vastly greater impact on targeted collective outcomes of computation. As for how the high-level properties of such a framework can be validated as being correct, that question is left for future exploration.

\section{General Use Case for Cognitive Computing}

Not all problems require general problem-solving ability. Computable problems are those that can be solved by deterministic algorithms. Any well-defined reasoning process can be automated, that is, can be represented by such an algorithm. Computable problems can be addressed by conventional computing methods as well as by cognitive computing. From the Human-Centric Functional Modeling point of view, intelligence involves a cognitive awareness process that provides the potential ability to navigate all possible reasoning processes. Where cognitive computing differs from conventional computing is that this cognitive awareness process also provides the ability to solve uncomputable problems through awareness of what the solutions can be observed to be. In the human mind, detecting solutions by observing past patterns is type 1 (fast) or intuitive reasoning, whereas computing solutions using methodical processes is type 2 (slow) or rational methodical reasoning. The cognitive awareness process combines both types of reasoning processes into a library that it can use to increase it's general problem-solving ability [12].

General problem-solving ability might be the most sustainable way to solve problems given finite resources. Even if the solution to a problem is computable, a given computing system might not have the resources to execute any specific computational algorithm. In that case, the solution is effectively uncomputable. However, if the system has general problem-solving ability, it can simply choose another algorithm that might not be able to determine the exact answer, but can compute some answer that is more correct than the current state of not having provided any answer at all. It's important to note that from the HCFM point of view, general problem-solving ability is the potential ability to navigate the entire conceptual space from any general problem to any general solution. Every living animal is an adaptive problem-solving system with one or more adaptive domains. General problemsolving ability is adaptive problem-solving in the cognitive domain. Human-like general problemsolving ability has been suggested to have specific well-defined properties [13]. But the resources for human-like general problem-solving ability are not necessary for all problems. An ant, for example, might not have sufficient intelligence to play chess, but it solves some general problems like finding food quite efficiently. From the HCFM point of view, the types of problems that a given cognitive system can solve are well-defined [14].

Cognitive communication can then potentially consist of swarms of very low intelligence agents that self-assemble into communication networks, collections of highly intelligent agents that select the algorithms by which they or the swarms under their control self-assemble into communication 
networks, or any combination of the two. Furthermore, the intelligence of agents might even be changed dynamically to suit changing resource constraints. In order to achieve all these possibilities it is necessary to define a single cognitive communication solution, and generalize to find all others.

\section{Cognitive Computing in Software and Hardware}

From the HCFM perspective, some processes involved in telecommunication are physical processes, and some are cognitive processes. Cognitive computing processes, including cognitive communication, can potentially be implemented in either software or hardware.

\section{Services Provider and Consumer Roles in Cognitive Communication}

Some processes involved in cognitive communication might occur within hardware or software owned by services providers, and others occur within hardware or software owned by the consumer. These distinctions are not necessarily fixed. What is the function of a consumer device owned by an individual might at some point become a shared service. In any industry, cognitive computing can occur all along the entire product or service life-cycle from design, to sales, to recycling. In telecommunications, cognitive computing can occur within shared services in the domain of service providers, and within individual services in the domain of consumers, as can collective cognitive computing.

Cognitive design processes are an example of biomimicry in that they attempt to mimic adaptive problem-solving in nature. In nature, adaptive problem-solving systems continually evolve by changing the boundaries of functional components, and by changing the functions involved in those components. From this perspective, services are subsystems which provide functions used by other parts of the system. Other functional components are just used locally. As a biological example, the digestive system is likely used by each cell in the human organism. But of course, in another organism, each cell in the organism might have it's own digestive system. The importance of this distinction between shared services and local functions of functional components is that it defines the boundaries between services providers and consumers in an individual or collective cognitive computing system.

\section{Cognitive Communication and Telecommunications Services}

If services are decoupled into components, a GCI can orchestrate the process of finding the optimal service provider to implement each component. A GCI can also orchestrate the process of finding chains of cooperation with local businesses that reliably result in powerful competitive advantage for local service providers to perform that implementation when they are available. In order to enable the group to reliably out-compete any centralized company, this approach has the potential to decentralize ownership so that benefit can be aligned with the interests of a much larger group, which in turn allows cooperation to be aligned with the larger set of activities belonging to that group. For example, rather than a single services provider deploying a great number of base stations to form a network, a single individual might own one base station, and the GCI will orchestrate development of a telecommunications platform that will automate the process of enabling each services provider to receive a portion of the revenue from the calls carried per month. This decentralized approach can enable more heterogeneous connectivity while also dramatically reducing cost for the consumer, and at the same time increasing benefit for the telecommunications provider that cooperates to implement such a system.

\section{Synergies Potentially Achievable by Services Providers through Cognitive Communication}

\section{Cost Reducing Synergies}

Modular hardware and software require only

partial upgrades.

\section{Profit Increasing Synergies}

Owners of base stations and other hardware with cognitive communication capabilities can potentially 
Economies of scale reduce cost of hardware and software through use of GCI to share designs, production, and to scale other cooperation.

The cost of offering services can be reduced by using GCI to algorithmically find synergies that reduce the cost of every required product or service, from financing, to hardware. Examples might be combining with local job creation programs to subsidize subcontractors, or combining with renewable energy projects to reduce power costs.

Reduce the cost of offering services through creating a more efficient market for procuring subcontracting services.

In a decentralized telecommunications model based on GCI a group of individuals might each purchase a single base station or other hardware with cognitive communication capabilities. This radically reduces the cost barrier to entering the telecommunications market.

The ability to use any available spectrum through techniques like cognitive radio creates the potential to lower profitability of existing telecom services providers until they might reliably be incentivized by such a collective to sell existing spectrum.

Table 1: Synergies potentially achievable for services providers through cognitive communication.

\section{Cognitive Communication and Consumer Electronics Hardware}

In hardware developed through a process with the capacity for collective design cognition, hardware functionality would be expected to be abstracted to create common functional models for all devices that communicate, where those models would allow functional components (modules) implementing those functions to achieve maximal impact on any outcome. For example, one outcome is a high service level, where service level includes properties such as maintainability. Devices are highly maintainable from the user-centric perspective when they are built in terms of modules replaceable by the end user with little to no specialized tools or expertise. Rather than having to buy a new cell phone or other device when the communication network changes, a more modular approach would allow the user to simply replace the communication module so that a phone might be useful for much longer. By making all parts modular, a phone might last a lifetime. However, the challenge is not that such phones cannot be made today. There are a number of modular phone designs that have been proposed by various startups. The challenge instead is that no company could expect to stay in business by selling only one phone to each individual in their lifetime. However, where the dollar value of cooperation is greater than zero, GCI has the potential to scale cooperation until the value of cooperation is large enough to provide competitive advantage for groups that cooperate. The solution is then to use GCI to 
cooperate to share designs of components across a sufficient number of devices, and to share production over a sufficient number of value chains, so that a business could quite comfortably sell a phone expected to last a lifetime.

\section{Cognitive Communication and Consumer Software Applications}

Any application that sends or receives data might be considered to be a communication application, such as text or video based instant messaging, email, web browsers, or content streaming services. From the functional modeling perspective, cognitive communication in software applications (cognitive computation in software in the communication domain) involves user interaction with an intelligent agent that executes such applications. This execution by an intelligent agent in turn involves defining functional models of applications, so that the intelligent agent can select whatever implementation of a given functional component is most fit at executing the required functionality in the current context. In this sense, software applications self-assemble according to the functionality required, the device form factor, and other factors. In the current smartphone environment there might be several applications that perform essentially the same messaging function within different social networks. In a cognitive communication device on the other hand, an intelligent agent might download the semantic model for the application, download semantic models for the various widgets that might occur, and then use GCI to self-assemble social networks to communicate through any functionality the user decides to communicate through. As an example, in the current communication environment an individual might desire to use the telephone to communicate over the Internet with a given individual who might only have a web browser and email installed. That communication would require the second individual installing the software and setting up a new account. In the cognitive communication environment, a smart agent working on behalf of the first user might determine all the channels through which the second person could be contacted, and then send a request to talk on the phone through one of those channels. A user agent working on behalf of the second user might accept or deny the request based on policies that user has defined. If the request is accepted, that agent will negotiate the initiation of the call with the other intelligent agent, and then execute that call through whatever telephone app functionality and user interface the second user is familiar with. The first user might see a different user interface. When new functionality is required, the intelligent agent could potentially insert it on the fly. By minimizing the software required to achieve a given function, cognitive computing has the potential to maximize performance per given hardware.

\section{Functional Modeling Tools Required for Cognitive Computing}

In order for a system of individual or collective cognitive computing to execute communication functions, semantic models of those functions must exist. An eXtensible Domain Modeling Framework (XDMF) [15] has been defined in the attempt to divide all the things a business can do into a number of subject areas or domains. For each domain XDMF uses HCFM to define a set of plain English "operations" describing what the business can do in this area. Having a closed set of operations (or in other words a closed set of notation elements) with which to describe (i.e. model) a given domain is a key element of Model-Driven Architecture. With such a Domain Specific Model, implementations of those elements create the possibility of round-trip engineering in which the model is used to generate software or designs for hardware that implement that functionality. And models can be generated to represent software or hardware implementing any given functionality.

Any ontology is likely to have the capacity to express only a subset of meaning, because while a set of operations on data can be shown to be Turing-complete, there is no similar set of operations universally agreed as being capable of spanning the entire set of cognitive operations that human's might use to express meaning. However, while incomplete, ontologies are nevertheless quite useful, and are necessary in cognitive computing. The challenge is not to get rid of ontologies, but to gain the ability to 
extend them in a dynamic and automated way to cover as high a proportion of meanings as is useful to do, within a more comprehensive semantic model that can represent all meaning [14].

\title{
Generalizing Cognitive Communication to Apply Elsewhere
}

Any industry in which processes can be automated and therefore executed by a system of cognitive computing, is an industry in which the cognitive computing model might be applied to optimize individual or collective outcomes. The process of applying cognitive computing is the same, define a set of functions that span the functional state space of businesses within the domain (e.g. the transportation domain), define processes by which businesses might navigate that functional state space (e.g. define transportation processes in terms of those transportation functions), and then define the AGI functionality that an intelligent agent might use to navigate individual processes in order to optimize outcomes for that individual business per unit of resources, and in addition define the GCI functionality that a group of businesses might use to navigate collective processes in order to optimize collective outcomes for the group per unit of resources. Since the methods by which outcomes are optimized might be independent of the problem being solved, this AGI and GCI functionality might well be common across a number of such implementations. The author to his knowledge has defined the only functional models of AGI and GCI with the potential capacity for general problem-solving ability and that are therefore suitable to be used as models of individual and collective cognitive computation. GCI is new, though prestigious institutes such as the MIT Center of Collective Intelligence have defined core concepts. Because it is new, examples exploring the application of this model of cognitive computing therefore by necessity refer to the authors own work.

\author{
Applying Cognitive Computing Across the Entire Business Life-Cycle of Product and Services \\ Cognitive Transportation \\ Cognitive Manufacturing [19] \\ Cognitive Shipping \\ Cognitive Customer Service \\ Cognitive Healthcare [16] \\ Cognitive Finance \\ Cognitive Agriculture [17] \\ Table 2: The above industries have been assessed as most likely to be impacted by automation. \\ Cognitive computing might therefore be applied in all of them.
}

The application of cognitive computing in all of these areas is currently being explored in a Collective Intelligence based Program to Accelerate Achievement of the Sustainable Development Goals (CIPAASDGs) [17]. The first phase of this program is intended to focus on agriculture.

Furthermore, cognitive computing based processes might be applied across the entire product and services business life-cycle.

\author{
Applying Cognitive Computing Across the Entire Business Life-Cycle of Product and Services \\ Cognitive Architecture [18] \\ Cognitive Design [19] \\ Cognitive Manufacturing [19] \\ Cognitive Marketing \\ Cognitive Sales \\ Cognitive Distribution \\ Cognitive Maintenance \\ Cognitive Recycling
}


Table 3: Application of cognitive computing across the entire product and services life-cycle.

Exploration of the application of cognitive computing to marketing, sales, and other processes is also ongoing.

\section{Conclusion}

From the functional modeling perspective systems of individual cognition are adaptive problemsolving systems that maximize individual outcomes per unit of resources. And systems of collective cognition are adaptive problem-solving systems that maximize collective outcomes per unit of resources. Systems of individual cognition might be implemented by intelligent agents. And systems of collective cognition might be implemented by GCI. Defining semantic models of all communication processes, and implementing intelligent agents to navigate individual communication processes, as well as implementing GCI to navigate collective communication processes has the potential to achieve higher levels of current outcomes that can currently be achieved, particularly security and sustainability, but also to achieve outcomes that are not reliably achievable otherwise, such as truly decentralized services. By defining use cases where cognitive computing might be used in other industries, the cost of implementing such semantic modeling tools and intelligent systems might be spread over many different projects so that it becomes feasible, where implementing such systems might not currently be economic for any one project.

\section{References}

[1] Williams, A. E. (2020, April 20). A Model for Artificial General Intelligence. https://doi.org/10.31730/osf.io/ xtq2V (in print in the proceedings of AGI 2020)

[2] The Relationship Between Collective Intelligence and One Model of General Collective Intelligence, Andy E. Williams, Computational Collective Intelligence, 11th International Conference, ICCCI 2019, Hendaye, France, September 4-6, 2019, Proceedings, Part II, Pages 589-600

[3] Williams, A. E. (2020, April 30). A Model for General Collective Intelligence.

https://doi.org/10.31730/osf.io/6u984

[4] Cui, C., Yang, D. \& Jin, S. Robust Spectrum-Energy Efficiency for Green Cognitive Communications. Mobile Netw Appl (2019). https://doi.org/10.1007/s11036-019-01347-y

[5] K. Yang, S. Liu, L. Cai, Y. Yilmaz, P. Chen and A. Walid, "Guest Editorial Special Issue on AI Enabled Cognitive Communication and Networking for IoT," in IEEE Internet of Things Journal, vol. 6, no. 2, pp. 19061910, April 2019, doi: 10.1109/JIOT.2019.2908443.

[6] David Chelmins, Janette Briones, Joseph Downey, Gilbert Clark, Adam Gannon, COGNITIVE COMMUNICATIONS FOR NASA SPACE SYSTEMS, retrieved from https://ntrs.nasa.gov/citations/20190032643, on Sept 14, 2020.

[7] Ehsan Tohidi, David Gesbert, Philippe Ciblat, Distributed Resource Allocation Algorithms for MultiOperator Cognitive Communication Systems, arXiv:2005.04001 [eess.SP]

[8] K. Thakkar, A. Goyal and B. Bhattacharyya, "Deep Learning and Channel Estimation," 2020 6th International Conference on Advanced Computing and Communication Systems (ICACCS), Coimbatore, India, 2020, pp. 745-751, doi: 10.1109/ICACCS48705.2020.9074414.

[9] Priya, B., Malhotra, J. QAAs: QoS provisioned artificial intelligence framework for AP selection in nextgeneration wireless networks. Telecommun Syst (2020). https://doi.org/10.1007/s11235-020-00710-9 [10] Andy E. Williams, General Collective Intelligence, Human-Centric Functional Modeling and the Current Limitations on Collective Design Cognition, working paper (2020)

[11] Andy E. Williams, General Collective Intelligence as the Emerging Paradigm in Design for Sustainable Development, in print, accepted to the proceedings of ISDRS 2020

[12] Williams, A. E. (2020, April 27). Defining Functional Models of Artificial Intelligence Solutions to Create a Library that an Artificial General Intelligence can use to Increase General Problem Solving Ability. https://doi.org/10.31730/osf.io/hpzb7 
[13] Williams, A. E. (2020, July 11). Human Intelligence and General Collective Intelligence as Phase Changes in Animal Intelligence. https://doi.org/10.31234/osf.io/dr8qn

[14] Andy E. Williams, Using Human-Centric Functional Modeling and a Functional Model of Cognition Towards Defining a Complete Model of Semantic Meaning, under review

[15] Andy E. Williams, The eXtensible Domain Modeling Framework, working paper (2020)

[16] Andy E. Williams, Re-imagining the Future of Healthcare with General Collective Intelligence, working paper (2020)

[17] Williams, A. E. (2020, April 30). The Collective Intelligence based Program to Accelerate Achievement of the Sustainable Development Goals as a Case Study for Collectively Intelligent Program Design. https://doi.org/10.31235/osf.io/r2dxq

[18] Andy E. Williams, A Proposed General Collective Intelligence Based Smart Sustainable Housing and Smart Cities Design Program, in print (Conference on Sustainable Architecture Planning and Urban Design, 2020)

[19] Williams, A. E. (2020, August 17). General Collective Intelligence as Biomimicry of Nature's Design and Manufacturing Processes. https://doi.org/10.31730/osf.io/92rqx 